WINPEC Working Paper Series No.E1419

January2015

Several bases of a game space and an application to the Shapley value

Koji Yokote, Yukihiko Funaki

Waseda INstitute of Political EConomy

Waseda University

Tokyo,Japan 


\title{
Several bases of a game space and an application to the Shapley value
}

\author{
Koji Yokote* Yukihiko Funaki ${ }^{\dagger}$
}

January 23, 2015

\begin{abstract}
We introduce several bases of the set of TU games. Given a coalition $T$, Yokote et al. (2013) introduced the commander game in which a coalition including 1 player in $T$ obtains payoff. On the other hand, Shapley (1953) introduced the unanimity game in which a coalition including all players in $T$ obtains payoff. We consider the intermediate between the two games. We introduce a game in which a coalition including $k$ players in $T$ obtains payoff, where $1 \leq k \leq|T|$. We show that, if there is a specific relationship between the size of coalition $T$ and $k$, we can construct a new basis. By using the new basis, we give sufficient conditions under which the Shapley value coincides with the prenucleolus.
\end{abstract}

JEL classification: C71

Keywords: basis; Shapley value; prenucleolus; coincidence condition

\section{Introduction}

This paper is a complement study to Yokote et al. (2013). Yokote et al. (2013) introduced a new basis that has two desirable properties. First, when we express a game by a linear combination of the basis, the coefficients related to singletons coincide with the Shapley value. Second, the basis induces the null space of the Shapley value. The basis consists of the commander game

*JSPS Research Fellow. Graduate School of Economics, Waseda University, 1-6-1, Nishi-Waseda, Shinjuku-ku, Tokyo 169-8050, Japan (sidehand@toki.waseda.jp)

${ }^{\dagger}$ Faculty of Political Science and Economics, Waseda University, 1-6-1, Nishi-Waseda, Shinjuku-ku, Tokyo 169-8050, Japan (funaki@waseda.jp) 
defined as follows: the commander game defined for $T$ assigns 1 to a coalition including 1 player in $T$. On the other hand, the well-known unanimity game (Shapley (1953)) is defined as follows: the unanimity game defined for $T$ assigns 1 to a coalition including all players in $T$. The above games represent two extreme cases: a coalition includes only 1 player, or all players. In this paper, we consider the intermediate between the two cases and introduce new bases.

We introduce the following game: the game defined for coalition $T$ assigns 1 to a coalition including $k$ players in $T$, where $1 \leq k \leq|T|$. We show that, if there is a specific relationship between the size of coalition $T$ and $k$, then we can construct a basis. This result includes the bases of the commander games and the unanimity games as special cases.

We focus on the basis that consists of the games with the following property: the game defined for coalition $T$ with even number of players assigns 1 to a coalition including half players in $T$. By using this basis, we give sufficient conditions under which the Shapley value coincides with the prenucleolus. We show that the games defined for coalitions with even number of players span the set of all games satisfying PS property, introduced by Kar et al. (2009). As they proved, under PS property, the Shapley value and the prenucleolus coincide. We also give new sufficient condition of the coincidence. The results enable us to construct a linear subspace where the coincidence holds. For other approaches to the subspace, see Chang and Tseng (2011).

This paper is organized as follows. In Section 2, we introduce notations and definitions. In Section 3, we prove that the set of new simple games constitutes the basis. In Section 4, we give sufficient conditions under which the Shapley value coincides with the prenucleolus by using the new basis.

\section{Preliminary}

A TU game is a pair of $(N, v)$, where $N$ is a finite set of $n$ players and $v$ is a function from $2^{N}$ to $\mathbb{R}$ and satisfies $v(\emptyset)=0$. The value $v(S), S \in 2^{N}$, represents the attainable payoff for players in $S$ when they cooperate. In the remaining part, we fix player set $N$. So, for simplicity, we write $v$ instead of $(N, v)$. Let $\Gamma$ denote the set of all games with player set $N$. For any two games $v, w \in \Gamma$ and $\alpha \in \mathbb{R}$, we define the sum and the scalar multiplication as follows: $(v+w)(S)=v(S)+w(S)$ for all $S \in 2^{N}$, and $(\alpha v)(S)=\alpha v(S)$ for all $S \in 2^{N}$. Then, we can identify the set $\Gamma$ as $2^{n}-1$ dimensional vector space. For a set of games $\left\{v^{k}\right\}_{k=1}^{m}, m \in \mathbb{N}, v^{k} \in \Gamma^{N}$ for all $k=1, \cdots, m$, we 
define the set spanned by the games as follows:

$$
\left.\operatorname{Sp}\left(\left\{v^{k}\right\}_{k=1}^{m}\right\}\right)=\left\{\sum_{k=1}^{m} \alpha^{k} v^{k}: \alpha^{k} \in \mathbb{R} \text { for all } k=1, \cdots, m\right\} .
$$

We consider a desirable rule that distributes the total attainable payoff $v(N)$. Such a rule is described as a solution function. A solution function $\psi$ assigns an $n$-dimensional payoff vector to each game $v \in \Gamma$. In the remaining part, we focus on the following two solution functions: the Shapley value and the prenucleolus. For a player $i \in N$, a game $v \in \Gamma$ and a coalition $S \subseteq N, i \notin S$, we define the marginal contribution of player $i$ to coalition $S$ as follows:

$$
\Delta_{i} v(S)=v(S \cup\{i\})-v(S) .
$$

The Shapley value, introduced by Shapley (1953), is defined as follows: for any $v \in \Gamma^{N}$,

$$
\phi_{i}(v)=\sum_{S \subseteq N: i \notin S} \frac{|S| !(n-|S|-1) !}{n !}(v(S)-v(S \backslash\{i\})) \text { for all } i \in N .
$$

For any $v \in \Gamma, S \subseteq N, S \neq \emptyset$, and $x \in \mathbb{R}^{n}$, we define the excess of coalition $S$ with respect to $x$ in $v$ as follows: $e(S, x, v)=v(S)-\sum_{i \in S} x_{i}$. For any $x$ and $y \in \mathbb{R}^{n}, y \geq_{\text {lex }} x$ means that $y$ is greater than $x$ in the lexicographic ordering of $\mathbb{R}^{n}$. Let $\theta(x)=\left(\theta_{1}(x), \theta_{2}(x), \ldots, \theta_{2^{n}-1}(x)\right) \in \mathbb{R}^{2^{n}-1}$ denote the sequence of excess of $S \subseteq N, S \neq \emptyset$, with respect to $x$, where $\theta_{t}(x) \geq \theta_{t+1}(x)$ for all $t, 1 \leq t \leq 2^{n}-2$. The prenucleolus $\nu$, introduced by Schmeidler (1969), is defined as follows: for any $v \in \Gamma^{N}$,

$$
\nu(v)=\left\{x \in I(v): \theta(y) \geq_{\text {lex }} \theta(x) \text { for all } y \in I(v)\right\}
$$

where

$$
I(v)=\left\{x \in \mathbb{R}^{n}: \sum_{i \in N} x_{i}=v(N) \text { for all } i \in N\right\}
$$

For a game $v \in \Gamma$ and $\beta \in \mathbb{R}^{n}$, we define $v+\beta$ by $(v+\beta)(S)=v(S)+\sum_{i \in S} \beta_{i}$ for all $S \subseteq N, S \neq \emptyset$. We say that a game $v$ is strategically equivalent to $w$ if there exists a vector $\beta \in \mathbb{R}^{n}$ such that $w=v+\beta$. For a game $v \in \Gamma^{N}$, player $i \in N$ is called a null player if $v(S \cup\{i\})-v(S)=0$ for all $S \subseteq N, i \notin S$. For a game $v \in \Gamma$ and a permutation $\pi$ of $N$, we define $\pi v$ by $\pi v(S)=v(\pi S)$ for all $S \subseteq N, S \neq \emptyset$. We give axioms satisfied by a solution function $\psi$.

Weak Strategic Invariance For any $v \in \Gamma^{N}$ and $\beta \in \mathbb{R}^{n}$, we have $\psi(v+$ $\beta)=\psi(v)+\beta$. 
Efficiency For any $v \in \Gamma^{N}, \sum_{i \in N} \psi_{i}(v)=v(N)$.

Null Player Property Let $v \in \Gamma^{N}$. If $i \in N$ is a null player, then $\psi_{i}(v)=$ 0 .

Symmetry Let $v \in \Gamma$ be a game and $\pi$ be a permutation of $N$ such that $v=\pi v$. Then, $\psi(v)=\pi \psi(v)$.

Both the Shapley value and the prenucleolus satisfy all axioms above.

\section{Extension of basis}

In Yokote et al. (2013), we introduced the commander game $\bar{u}_{T}$ for each coalition $T \in 2^{N} \backslash \emptyset$. The game $\bar{u}_{T}$ is defined as follows: $\bar{u}_{T}(S)=1$ if $|S \cap T|=$ 1 and 0 otherwise. On the other hand, the well-known unanimity game $u_{T}$, introduced by Shapley (1953), is defined as follows: $u_{T}(S)=1$ if $|S \cap T|=|T|$ and 0 otherwise. The two games capture two extreme cases. In the game $\bar{u}_{T}$, only one player in $T$ yields payoff, while in the game $u_{T}$, cooperation of all players in $T$ yields payoff. We consider the intermediate between the two extreme cases.

Let $T \subseteq N, k \in \mathbb{N}, 1 \leq k \leq|T|$. We define $\bar{u}_{T}^{k}$ by

$$
\bar{u}_{T}^{k}(S)= \begin{cases}1 & \text { if }|S \cap T|=k \\ 0 & \text { otherwise }\end{cases}
$$

Note that $\bar{u}_{T}^{1}=\bar{u}_{T}$ and $\bar{u}_{T}^{|T|}=u_{T}$.

Shapley (1953) and Yokote et al. (2013) respectively proved that the set $\left\{u_{T}\right\}_{T \in 2^{N} \backslash \emptyset}$ and $\left\{\bar{u}_{T}\right\}_{T \in 2^{N} \backslash \emptyset}$ are bases of $\Gamma$. In this section, we show that we can also construct a basis by using the game $\bar{u}_{T}^{k}$. In order to do that, we need a specific relationship between the size of coalition $T$ and the number of intersection $k$. Let us introduce a function that is used to describe the relationship. Consider a function $l:\{1, \cdots, n\} \rightarrow\{1, \cdots, n\}$ satisfying the following conditions:

C1: $l(1)=1$.

C2: $l(t)=l(t-1)$ or $l(t-1)+1$ or $l(t-1)-1$ for all $t=2, \cdots, n$.

We have the following result:

Theorem 1 Let $l$ be a function which satisfies $C 1$ and C2. Then, the set of games $\left\{\bar{u}_{T}^{l(|T|)}\right\}_{\emptyset \neq T \subseteq N}$ is a basis of $\Gamma$. 
Theorem 1 generalizes the result by Shapley (1953) and Yokote et al. (2013) in that both sets $\left\{u_{T}\right\}_{T \in 2^{N} \backslash \emptyset}$ and $\left\{\bar{u}_{T}\right\}_{T \in 2^{N} \backslash \emptyset}$ can be expressed by $\left\{\bar{u}_{T}^{l(|T|)}\right\}_{\emptyset \neq T \subseteq N}$ with a function $l$ satisfying $\mathrm{C} 1$ and $\mathrm{C} 2$. Before giving a precise proof, we explain the outline. The proof highly relies on the following lemma saying that the game $\bar{u}_{T}^{k}$ can be expressed by the addition of games with the superscript $k-1$ :

Lemma 1 Let $T \subseteq N,|T| \geq 2, k \in \mathbb{N}, 2 \leq k \leq|T|$. Then, we have

$$
\bar{u}_{T}^{k}=\frac{1}{k}\left(\sum_{i \in T} \bar{u}_{T \backslash\{i\}}^{(k-1)}-(|T|-k+1) \bar{u}_{T}^{(k-1)}\right) .
$$

For the proof of this lemma, see Appendix. The proof of Theorem 1 proceeds by induction. First, it is already proved that the set $\left\{\bar{u}_{T}^{1}\right\}_{T \in 2^{N} \backslash \emptyset}$ is a basis. Given this inductive base, we increase the sum of the number of superscripts. The inductive step is completed by using Lemma 1 .

Proof of Theorem 1. We introduce three notations with respect to the function $l$.

$$
\begin{aligned}
K(l) & =\sum_{k=1}^{n} l(k), \\
M(l) & =\max \{l(k): k=1, \cdots, n\}, \\
Q(l) & =\{k: l(k)=M(l)\} .
\end{aligned}
$$

If $K(l)=n$, then $l(k)=1$ for all $k=1, \cdots, n$, the proof is completed by the result of Yokote (2013). We establish the inductive step. Suppose that the result holds for all $l$ such that $l$ satisfies $\mathrm{C} 1, \mathrm{C} 2$, and $n \leq K(l) \leq p$. We prove the result for $K(l)=p+1$, where $n \leq p \leq \frac{n(n+1)}{2}-1$.

Assume, by way of contradiction, that the set of games $\bar{u}_{T}^{l(|T|)}, K(l)=$ $p+1$, is not a basis. Then, there exists $\left(\lambda_{T}\right)_{\emptyset \neq T \subseteq N} \neq \mathbf{0}$ such that

$$
\sum_{T \subseteq N: T \neq \emptyset} \lambda_{T} \bar{u}_{T}^{l(|T|)}=\mathbf{0}
$$

Let $q \geq 2$ denote the natural number such that $l(q)=M(l)$ and $q \leq k$ for all $k \in Q(l)$. Then, the following equation holds:

$$
l(q-1)=l(q)-1 \geq 1 .
$$

From equation (1), we have

$$
\sum_{T \subseteq N: T \neq \emptyset,|T| \neq q} \lambda_{T} \bar{u}_{T}^{l(|T|)}+\sum_{S \subseteq N:|S|=q} \lambda_{S} \bar{u}_{S}^{l(q)}=\mathbf{0} .
$$


By applying Lemma 1, we have

$$
\begin{aligned}
& \sum_{T \subseteq N: T \neq \emptyset,|T| \neq q} \lambda_{T} \bar{u}_{T}^{l(|T|)} \\
+ & \sum_{S \subseteq N:|S|=q} \frac{\lambda_{S}}{l(q)}\left(\sum_{i \in S} \bar{u}_{S \backslash\{i\}}^{(l(q)-1)}-(q-l(q)+1) \bar{u}_{S}^{(l(q)-1)}\right)=\mathbf{0} .
\end{aligned}
$$

We define $l^{\prime}$ by

$$
l^{\prime}(|T|)= \begin{cases}l(|T|) & \text { if }|T| \neq q \\ l(q)-1 & \text { if }|T|=q .\end{cases}
$$

We show that $l^{\prime}$ satisfies $\mathrm{C} 1$ and $\mathrm{C} 2$. Since $q \geq 2, l^{\prime}(1)=l(1)=1$, which proves $\mathrm{C} 1$. Since $l(q)=M(l)$, we have

$$
l(q+1)=l(q) \text { or } l(q)-1 .
$$

If $l(q+1)=l(q)$, then $l^{\prime}(q+1)=l(q+1)=l^{\prime}(q)+1$. If $l(q+1)=l(q)-1$, then $l^{\prime}(q+1)=l(q+1)=l^{\prime}(q)$. Namely, $l^{\prime}(q+1)=l^{\prime}(q)+1$ or $l^{\prime}(q)$. In addition, $l^{\prime}(q)=M(l)-1=l(q-1)=l^{\prime}(q-1)$, which proves $\mathrm{C} 2$.

By using the function $l^{\prime}$, equation (2) can be written as follows:

$$
\begin{aligned}
& \sum_{T \subseteq N: T \neq \emptyset,|T| \neq q} \lambda_{T} \bar{u}_{T}^{l^{\prime}(|T|)} \\
+ & \sum_{S \subseteq N: S \neq \emptyset,|S|=q} \frac{\lambda_{S}}{l(q)}\left(\sum_{i \in S} \bar{u}_{S \backslash\{i\}}^{l^{\prime}(|S \backslash\{i\}|)}-\left(q-l^{\prime}(q)\right) \bar{u}_{S}^{l^{\prime}(q)}\right)=\mathbf{0 .} .
\end{aligned}
$$

Since $K\left(l^{\prime}\right) \leq p$, equation (3) contradicts the induction hypothesis.

As Yokote et al. (2013) proved, the set $\left\{\bar{u}_{T}^{1}\right\}_{\emptyset \neq T \subseteq N}$ has two desirable properties. First, when we express a game $v$ by a linear combination of the basis, the coefficients related to singletons coincide with the Shapley value. Second, the set of games defined for coalitions with no less than 2-players spans the null space of the Shapley value. In order that the new basis $\left\{\bar{u}_{T}^{l(|T|)}\right\}_{T \in 2^{N} \backslash N}$ has the same property, we need additional conditions on the function $l$. The important fact here is that, in order to induce the null space of the Shapley value, the worth of the grand coalition in the game $\bar{u}_{T}^{l(|T|)}, T \subseteq N,|T| \geq 2$, must be equal to 0 . In other words, we must have $l(|T|)<|T|$ for $T \subseteq N$, $|T| \geq 2$. So, we consider a function $l:\{1, \cdots, n\} \rightarrow\{1, \cdots, n\}$ which satisfies the following conditions:

$\mathrm{C} 1^{\prime}: l(1)=l(2)=1$, 
C2': $l(t)=l(t-1)$ or $l(t-1)+1$ or $l(t-1)-1$ for all $t=3, \cdots, n$.

Theorem 2 Let $l$ be a function which satisfies $C 1^{\prime}$ and $C 2^{\prime}$. Then,

1: The set of games $\left\{\bar{u}_{T}^{l(|T|)}\right\}_{\emptyset \neq T \subseteq N}$ is a basis of $\Gamma$.

2: When we express a game $v \in \Gamma$ by a linear combination of this basis, the coefficient of $\bar{u}_{\{i\}}^{1}$ is equal to $\phi_{i}(v)$ for all $i \in N$.

3: The set $\left\{\bar{u}_{T}^{l(|T|)}: T \subseteq N,|T| \geq 2\right\}$ spans the null space of $\phi$.

Proof. The set is a basis from Theorem 1. Let $T \subseteq N,|T| \geq 2$, and $j \in N \backslash T$. Then, for any $S \subseteq N \backslash\{j\}$, we have $|S \cap T|=|(S \cup\{j\}) \cap T|$. It follows that $j$ is a null player, and we obtain $\phi_{j}\left(\bar{u}_{T}^{l(|T|)}\right)=0$. From symmetry and the fact that $\bar{u}_{T}^{l(|T|)}(N)=0$, we have $\phi_{i}\left(\bar{u}_{T}^{l(|T|)}\right)=0$ for all $i \in N$. As a result, 3 holds. It remains to prove 2 . Let $v \in \Gamma$ be given. Let $\left(\alpha_{T}\right)_{T \in 2^{N} \backslash \emptyset}$ denote the coefficients in the linear combination representing $v$ by $\bar{u}_{T}^{l(|T|)}$. Then, for any $i \in N$,

$$
\begin{aligned}
\phi_{i}(v) & =\phi_{i}\left(\sum_{T \in 2^{N} \backslash \emptyset} \alpha_{T} \bar{u}_{T}^{l(|T|)}\right) \\
& =\sum_{T \in 2^{N} \backslash \emptyset} \alpha_{T} \phi_{i}\left(\bar{u}_{T}^{f(|T|)}\right) \\
& =\sum_{j \in N} \alpha_{\{j\}} \phi_{i}\left(\bar{u}_{\{j\}}^{1}\right) \\
& =\alpha_{\{i\}},
\end{aligned}
$$

where the fourth equality holds from $\phi_{i}\left(\bar{u}_{\{i\}}^{1}\right)=1$ and $\phi_{i}\left(\bar{u}_{\{j\}}^{1}\right)=0$ for all $j \in N \backslash\{i\} .^{1}$

\section{Basis and coincidence condition}

The purpose of this section is to give sufficient conditions under which the Shapley value coincides with the prenucleolus by using the basis introduced in Section 3.

Let us consider the following function $f: 2^{N} \rightarrow \mathbb{N}$ :

$$
f(T)= \begin{cases}1 & \text { if }|T|=1, \\ \frac{|T|+1}{2} & \text { if }|T| \text { is an odd number, }|T| \geq 2 \\ \frac{|T|}{2} & \text { if }|T| \text { is an even number, }|T| \geq 2 .\end{cases}
$$

\footnotetext{
${ }^{1}$ These equations immediately follow from null player property and efficiency.
} 
For a coalition $T$ with even number of players, the game $\bar{u}_{T}^{f(T)}$ captures the situation in which a coalition including half players in $T$ obtains payoff. From Theorem 1, the set of games $\left\{\bar{u}_{T}^{f(T)}\right\}_{\emptyset \neq S \subseteq N}$ is a basis. Thus, any game $v \in \Gamma$ can be represented by a linear combination of the basis. Let $q(T, v)$ denote the coefficients in the linear combination, that is,

$$
v=\sum_{T \in 2^{N} \backslash \emptyset} q(T, v) \bar{u}_{T}^{f(T)} .
$$

For any $v \in \Gamma$, we define $v^{S h}$ by

$$
v^{S h}=v-\sum_{i \in N} q(\{i\}, v) \bar{u}_{\{i\}}^{1}
$$

Note that, from Theorem 2,

$$
\begin{aligned}
\phi\left(v^{S h}\right) & =\phi(v)-\sum_{i \in N} q(\{i\}, v) \phi\left(\bar{u}_{\{i\}}^{1}\right) \\
& =\phi(v)-\sum_{i \in N} \phi_{i}(v) \phi\left(\bar{u}_{\{i\}}^{1}\right)=\mathbf{0} .
\end{aligned}
$$

The game $v^{S h}$ is called the excess game with respect to the Shapley value. For any $v \in \Gamma$, we define

$$
\mathcal{Q}(v)=\left\{T \in 2^{N} \backslash \emptyset: q(T, v) \neq 0,|T| \geq 2\right\} .
$$

We have the following result:

Theorem 3 Let $v \in \Gamma$. Then, $\phi(v)=\nu(v)$ if at least one of the following conditions holds:

Condition 1: $T, T^{\prime} \in 2^{N} \backslash \emptyset$ and $|T|=\left|T^{\prime}\right| \geq 2$ imply $q(T, v)=q\left(T^{\prime}, v\right)$.

Condition 2: $T \in \mathcal{Q}(v)$ implies $|T|$ is an even number.

Condition 3: $T, T^{\prime} \in \mathcal{Q}(v)$ and $T \neq T^{\prime}$ imply $|T|=\left|T^{\prime}\right|$ and $T \cap T^{\prime}=\emptyset$.

We divide the remaining part into three subsections and prove each condition of Theorem 3. It will be proved that Condition 1 is equivalent to saying that that game $v$ is strategically equivalent to a symmetric game, and Condition 2 is equivalent to PS property introduced by Kar et al. (2009). Condition 3 is a new condition in this paper. 


\subsection{Proof of Condition 1}

We prove that $v$ is strategically equivalent to a symmetric game if and only if $v$ satisfies Condition 1 . We say that a game $v \in \Gamma$ is symmetric if

$$
|S|=|T| \text { implies } v(S)=v(T) \text { for all } S, T \subseteq N .
$$

From Symmetry, the coincidence between $\phi$ and $\nu$ immediately follows in symmetric games.

Proposition 1 Let $v \in \Gamma$. Then, $v$ is strategically equivalent to a symmetric game if and only if $v$ satisfies Condition 1.

Proof. Let $k \in \mathbb{N}, 1 \leq k \leq n$. We define the game $\bar{u}^{k}$ by

$$
\bar{u}^{k}=\sum_{T \subseteq N:|T|=k} \bar{u}_{T}^{f(T)} .
$$

Let $\bar{k}=f(T)$, where $T$ is a coalition such that $|T|=k$. In this game, we have

$$
\bar{u}^{k}(S)= \begin{cases}\left(\begin{array}{c}
|S| \\
\bar{k}
\end{array}\right) \cdot\left(\begin{array}{c}
|N \backslash S| \\
k-\bar{k}
\end{array}\right) & \text { if }|S| \geq \bar{k}, \\
0 & \text { if } 1 \leq|S|<\bar{k} .\end{cases}
$$

It follows that $\bar{u}^{k}$ is a symmetric game. The set of symmetric games is a linear subspace of $\mathbb{R}^{2^{n}-1}$, and the dimension is equal to $n$. Since the set of games $\left\{\bar{u}^{k}\right\}_{k=1}^{n}$ is linearly independent, the set spans the space. As a result, we obtain the following statement:

A game $v$ is symmetric if and only if $T, T^{\prime} \in 2^{N} \backslash \emptyset$ and $|T|=\left|T^{\prime}\right|$ imply $q(T, v)=q\left(T^{\prime}, v\right)$.

Now, the change in the coefficients $q(\{i\}, v), i \in N$, corresponds strategically equivalent transformation, so the proof is completed.

\subsection{Proof of Condition 2}

We prove that Condition 2 is equivalent to PS property introduced by Kar et al. (2009). Let us review the definition. A game $v \in \Gamma$ satisfies PS property if the following condition is satisfied: for any $i \in N$, there exists $c_{i} \in \mathbb{R}$ such that

$$
\Delta_{i} v(S)+\Delta_{i} v(N \backslash(S \cup\{i\}))=c_{i} \text { for all } S \subseteq N \backslash\{i\} .
$$


The condition says that, for any player $i$, the sum of marginal contributions to a coalition and its complement is always constant.

We have the following result:

Proposition 2 Let $v \in \Gamma$. Then, $v$ satisfies $P S$ property if and only if $v$ satisfies Condition 2.

Before giving a proof, we briefly explain why this proposition holds. The statement of Condition 2 raises the following question: why the result holds only for even number. In order to answer this question, let us take a coalition $T \in 2^{N} \backslash \emptyset$ where $|T|$ is an even number, and go back to the definition of $\bar{u}_{T}^{f(T)}$. The game assigns 1 to a coalition $S$ if $S$ includes half players of $T$. Note that, for any coalition $S \in 2^{N}$, we have the following: $|S \cap T|=\frac{|T|}{2}$ if and only if $|(N \backslash S) \cap T|=\frac{|T|}{2}$. It follows that

$$
\bar{u}_{T}^{f(|T|)}(S)=\bar{u}_{T}^{f(|T|)}(N \backslash S) \text { for all } S \in 2^{N} .
$$

It is worth mentioning that if $|T|$ is an odd number, then the above equality does not necessarily hold. On the other hand, PS property can be characterized as follows:

Lemma 2 Let $v \in \Gamma$. Then, $v$ satisfies PS property if and only if $v^{S h}(T)=$ $v^{S h}(N \backslash T)$ for all $T \in 2^{N}$.

For the proof, see Appendix.

Let us give an additional notation. For a coalition $T \in 2^{N} \backslash \emptyset$, we define $e_{T, N \backslash T} \in \Gamma^{N}$ by

$$
e_{T, N \backslash T}(S)= \begin{cases}1 & \text { if } S=T \text { or } N \backslash T \\ 0 & \text { otherwise }\end{cases}
$$

We can easily check that the following equation holds: for any $T \in 2^{N} \backslash \emptyset$,

$$
\phi\left(e_{T, N \backslash T}\right)=\mathbf{0} .
$$

We now give a precise proof.

Proof of Proposition 2. Let $\Gamma^{P S}$ denote the set of all games which satisfy PS property. Then, from Lemma 2,

$$
\Gamma^{P S}=\left\{v \in \Gamma: v^{S h}(T)=v^{S h}(N \backslash T) \text { for all } T \in 2^{N}\right\} .
$$


We define the set of games $\Gamma^{Q}$ by $\Gamma^{Q}=\operatorname{Sp}\left(\left\{\bar{u}_{T}^{f(T)}: T \in 2^{N},|T| \geq 2,|T|\right.\right.$ is an even number $\left.\} \cup\left\{\bar{u}_{\{i\}}^{1}: i \in N\right\}\right)$.

Our final goal is to prove that $\Gamma^{P S}=\Gamma^{Q}$.

Take an arbitrary player $k \in N$ and fix. We define

$$
\Gamma^{*}=\operatorname{Sp}\left(\left\{e_{T, N \backslash T}: T \in 2^{N} \backslash \emptyset, k \notin T\right\} \cup\left\{\bar{u}_{\{i\}}^{1}: i \in N\right\}\right) .
$$

Claim 1 The set of games $\left\{e_{T, N \backslash T}: T \in 2^{N} \backslash \emptyset, k \notin T\right\} \cup\left\{\bar{u}_{\{i\}}^{1}: i \in N\right\}$ is linearly independent.

Proof. Take two vectors $\left(\beta_{T}\right)_{T \in 2^{N} \backslash \emptyset, k \notin T}$ and $\left(\alpha_{\{i\}}\right)_{i \in N}$ such that

$$
\sum_{T \in 2^{N} \backslash \emptyset, k \notin T} \beta_{T} e_{T, N \backslash T}+\sum_{i \in N} \alpha_{\{i\}} \bar{u}_{\{i\}}^{1}=\mathbf{0} .
$$

From linearity of the Shapley value,

$$
\begin{aligned}
\sum_{T \in 2^{N} \backslash \emptyset, k \notin T} \beta_{T} \phi\left(e_{T, N \backslash T}\right) & =-\sum_{i \in N} \alpha_{\{i\}} \phi\left(\bar{u}_{\{i\}}^{1}\right), \\
\mathbf{0} & =-\alpha .
\end{aligned}
$$

By substituting equation (7) to equation (6), we have

$$
\sum_{T \in 2^{N} \backslash \emptyset, k \notin T} \beta_{T} e_{T, N \backslash T}=\mathbf{0},
$$

which implies that $\left(\beta_{T}\right)_{T \in 2^{N} \backslash \emptyset, k \notin T}=\mathbf{0}$.

Claim $2 \Gamma^{P S}=\Gamma^{*}$.

Proof. Proof of $\Gamma^{P S} \subseteq \Gamma^{*}$ : Take an arbitrary game $v \in \Gamma^{P S}$. Then, $v^{S h}(T)=v^{S h}(N \backslash T)$, which implies that $v^{S h} \in \operatorname{Sp}\left(\left\{e_{T, N \backslash T}: T \in 2^{N} \backslash \emptyset, k \notin\right.\right.$ $T\})$. Since

$$
v=\sum_{i \in N} q(\{i\}, v) \bar{u}_{\{i\}}^{1}+v^{S h}
$$

we obtain $v \in \Gamma^{*}$.

Proof of $\Gamma^{*} \subseteq \Gamma^{P S}$ : Take an arbitrary game $v \in \Gamma^{*}$. Then, from Claim 1, there exists a unique vector $\left(\beta_{T}\right)_{T \in 2^{N} \backslash \emptyset, k \notin T},\left(\alpha_{\{i\}}\right)_{i \in N}$ such that

$$
v=\sum_{T \in 2^{N} \backslash \emptyset, k \notin T} \beta_{T} e_{T, N \backslash T}+\sum_{i \in N} \alpha_{\{i\}} \bar{u}_{\{i\}}^{1} .
$$


From Linearity of the Shapley value and equation (5), we have

$$
\phi\left(\sum_{T \in 2^{N} \backslash \emptyset, k \notin T} \beta_{T} e_{T, N \backslash T}\right)=\sum_{T \in 2^{N} \backslash \emptyset, k \notin T} \beta_{T} \phi\left(e_{T, N \backslash T}\right)=\mathbf{0} .
$$

From Theorem 2, the set of games $\left\{\bar{u}_{T}^{f(T)}: T \in 2^{N},|T| \geq 2\right\}$ spans the null space. So, there exists a unique vector $\left(\gamma_{T}\right)_{T \in 2^{N},|T| \geq 2}$ such that

$$
\sum_{T \in 2^{N} \backslash \emptyset, k \notin T} \beta_{T} e_{T, N \backslash T}=\sum_{T \in 2^{N}:|T| \geq 2} \gamma_{T} \bar{u}_{T}^{f(T)} .
$$

By substituting equation (9) to equation (8), we obtain

$$
v=\sum_{T \in 2^{N}:|T| \geq 2} \gamma_{T} \bar{u}_{T}^{f(T)}+\sum_{i \in N} \alpha_{\{i\}} \bar{u}_{\{i\}}^{1} .
$$

Since the set of games $\left\{\bar{u}_{T}^{f(T)}: T \in 2^{N} \backslash \emptyset\right\}$ is a basis of $\Gamma$, we must have

$$
\alpha_{\{i\}}=q(\{i\}, v) .
$$

By substituting equation (10) to equation (8), we have

$$
\begin{aligned}
v & =\sum_{T \in 2^{N} \backslash \emptyset, i \notin T} \beta_{T} e_{T, N \backslash T}+\sum_{i \in N} q(\{i\}, v) \bar{u}_{\{i\}}^{1}, \\
v^{S h} & =\sum_{T \in 2^{N} \backslash \emptyset, i \notin T} \beta_{T} e_{T, N \backslash T} .
\end{aligned}
$$

It follows that $v^{S h}(T)=v^{S h}(N \backslash T)$ for all $T \in 2^{N}$. From Lemma $2, v \in \Gamma^{P S}$.

From Claim 2, our final goal is to prove that $\Gamma^{*}=\Gamma^{Q}$. Clearly, $\Gamma^{Q} \subseteq \Gamma^{*}$ holds. In order to prove that the converse set-inclusion holds, we prove that $\operatorname{dim} \Gamma^{*}=\operatorname{dim} \Gamma^{Q}$.

$$
\begin{aligned}
\operatorname{dim} \Gamma^{P S} & =\left|\left\{T \in 2^{N} \backslash \emptyset: k \notin T\right\}\right|+n \\
& =\left|\left\{T \in 2^{N \backslash k} \backslash \emptyset\right\}\right|+n \\
& =\mid\left\{T \in 2^{N \backslash k} \backslash \emptyset:|T| \text { is an even number }\right\} \mid \\
& +\mid\left\{T \in 2^{N \backslash k} \backslash \emptyset:|T| \text { is an odd number }\right\} \mid+n \\
& =\mid\left\{T \in 2^{N} \backslash \emptyset:|T| \text { is an even number, } k \notin T\right\} \mid \\
& +\mid\left\{T \in 2^{N} \backslash \emptyset:|T \cup k| \text { is an even number, } k \notin T\right\} \mid+n \\
& =\operatorname{dim} \Gamma^{Q} .
\end{aligned}
$$




\subsection{Proof of Condition 3}

In order to prove Condition 3, we give some preliminaries. For a subset $\mathcal{T} \subseteq 2^{N} \backslash \emptyset$, we define $\chi_{\mathcal{T}}$ by

$$
\chi_{\mathcal{T}}(S)= \begin{cases}1 & \text { if } S \in \mathcal{T} \\ 0 & \text { otherwise }\end{cases}
$$

We define Shapley collection introduced by Chang and Tseng (2009). We say that a subset $\mathcal{T} \subseteq 2^{N} \backslash \emptyset$ is a Shapley collection if the following condition is satisfied:

$$
\phi\left(\chi_{\mathcal{T}}\right)=\mathbf{0}
$$

For a coalition $T \in 2^{N} \backslash \emptyset$, we define

$$
\mathcal{C}(T)= \begin{cases}\left\{S \in 2^{N} \backslash \emptyset:|T \cap S|=\frac{|T|}{2}\right\} & \text { if }|T| \text { is an even number, } \\ \left\{S \in 2^{N} \backslash \emptyset:|T \cap S|=\frac{|T|+1}{2}\right\} & \text { if }|T| \text { is an odd number. }\end{cases}
$$

The following lemma holds:

Lemma 3 Let $T_{1}, T_{2}, \cdots, T_{k} \in 2^{N} \backslash \emptyset$ be coalitions such that $T_{l} \cap T_{m}=\emptyset$ and $\left|T_{l}\right|=\left|T_{m}\right|$ for all $l, m=1, \cdots, k, l \neq m$. Then, $\cap_{l=1}^{k} \mathcal{C}\left(T_{l}\right)$ is a Shapley collection.

Proof. Let $\mathcal{T}=\cap_{l=1}^{k} \mathcal{C}\left(T_{l}\right)$ and $t=f\left(T_{l}\right), l=1, \cdots, k$. Then, $\chi_{\mathcal{T}}$ is the following game:

$$
\chi_{\mathcal{T}}(S)= \begin{cases}1 & \text { if }\left|S \cap T_{l}\right|=t \text { for all } l=1, \cdots, k . \\ 0 & \text { otherwise. }\end{cases}
$$

Take any two coalitions $T_{i}$ and $T_{j}, i \neq j$. Let $i^{\prime} \in T_{i}$ and $j^{\prime} \in T_{j}$. Since $T_{i} \cap T_{j}=\emptyset$, we have $i^{\prime} \neq j^{\prime}$. Consider a permutation $\pi$ on $N$ which satisfies the following conditions:

$$
\begin{aligned}
\pi\left(i^{\prime}\right) & =j^{\prime}, \pi\left(T_{i}\right)=T_{j}, \pi\left(T_{j}\right)=T_{i}, \\
\pi\left(T_{l}\right) & =T_{l} \text { for all } l=1, \cdots, k, l \neq i, h \neq j .
\end{aligned}
$$

Note that the permutation $\pi$ is well-defined since $\left|T_{i}\right|=\left|T_{j}\right|$. Let $S \in 2^{N}$. The following equations are equivalent:

$$
\begin{aligned}
\chi_{\mathcal{T}}(S) & =1, \\
\left|S \cap T_{l}\right| & =t \text { for all } l=1, \cdots, k, \\
\left|\pi(S) \cap \pi\left(T_{l}\right)\right| & =t \text { for all } l=1, \cdots, k, \\
\left|\pi(S) \cap T_{l}\right| & =t \text { for all } l=1, \cdots, k, \\
\chi_{\mathcal{T}}(\pi(S)) & =1 .
\end{aligned}
$$


It follows that for any $S \in 2^{N}$, we have $\chi_{\mathcal{T}}(S)=\chi_{\mathcal{T}}(\pi S)$. From Symmetry, $\phi_{i^{\prime}}\left(N, \chi_{\mathcal{T}}\right)=\phi_{j^{\prime}}\left(N, \chi_{\mathcal{T}}\right)$. Since $i^{\prime}$ and $j^{\prime}$ are arbitrary players, for any $i^{\prime}, j^{\prime} \in$ $\cup_{i=1}^{k} T_{i}$, we have $\phi_{i^{\prime}}\left(N, \chi_{\mathcal{T}}\right)=\phi_{j^{\prime}}\left(N, \chi_{\mathcal{T}}\right)$. Efficiency completes the proof.

We define three families of subsets of $2^{N} \backslash N$.

$\mathcal{P}=\left\{\mathcal{T} \subseteq 2^{N} \backslash N: \mathcal{T}=\mathcal{C}(T)\right.$ for some $\left.T \in \mathcal{Q}(v)\right\}$

$\mathcal{R}=\left\{\mathcal{T} \subseteq 2^{N} \backslash N: \mathcal{T}=\cap_{i=1}^{l} \mathcal{T}_{i}\right.$, where $\mathcal{T}_{i} \in \mathcal{P}$ for all $\left.i=1, \cdots, l, 1 \leq l \leq|\mathcal{Q}(v)|\right\}$,

$\mathcal{S}=\left\{\mathcal{T} \subseteq 2^{N} \backslash N: \mathcal{T}\right.$ is a Shapley collection $\}$

In the remaining part, we introduce some notations in measure theory. Let $X$ be a set and $\mathcal{X}$ be a family of subsets of $X$. We say that $\mathcal{X}$ is a Dynkin class if the following three conditions are satisfied:

$1 X \in \mathcal{X}$.

$\mathbf{2}$ Let $\mathcal{T}, \mathcal{T}^{\prime} \in \mathcal{X}$ be sets such that $\mathcal{T} \subseteq \mathcal{T}^{\prime}$. Then $\mathcal{T}^{\prime} \backslash \mathcal{T} \in \mathcal{X}$.

3 Let $\left\{\mathcal{T}_{k}\right\}_{k=1}^{\infty}$ be a family of disjoint sets in $\mathcal{X}$. Then, $\cup_{k=1}^{\infty} \mathcal{T}_{k} \in \mathcal{X}$.

Lemma $4 \mathcal{S}$ is a Dynkin class.

Proof. Step 1: From symmetry,

$$
\phi\left(\chi_{2^{N} \backslash N}\right)=\mathbf{0}
$$

It follows that $2^{N} \backslash N \in \mathcal{S}$.

Step 2: Suppose that $\mathcal{T}, \mathcal{T}^{\prime} \in \mathcal{S}, \mathcal{T} \subseteq \mathcal{T}^{\prime}$. From linearity,

$$
\phi\left(\chi_{\mathcal{T}^{\prime} \backslash \mathcal{T}}\right)=\phi\left(\chi_{\mathcal{T}^{\prime}}\right)-\phi\left(\chi_{\mathcal{T}}\right)=\mathbf{0}
$$

It follows that $\mathcal{T}^{\prime} \backslash \mathcal{T} \in \mathcal{S}$.

Step 3: Let $\left\{\mathcal{T}_{k}\right\}_{k=1}^{\infty}$ be a family of disjoint sets in $\mathcal{S}$. We define $\mathcal{T}=\cup_{k=1}^{\infty} \mathcal{T}_{k}$.

From linearity,

$$
\phi\left(\chi_{\mathcal{T}}\right)=\sum_{k=1}^{\infty} \phi\left(\chi_{\mathcal{T}_{k}}\right)=\mathbf{0}
$$

It follows that $\mathcal{T} \in \mathcal{S}$.

Let $\sigma(\mathcal{P})$ denote the $\sigma$-algebra generated from $\mathcal{P}$. Let $\mathcal{D}(\mathcal{R})$ denote the Dynkin class generated from $\mathcal{R}$. With respect to Dynkin class, the following result is known: 
Remark 1 (Dynkin's $\pi-\lambda$ theorem) Let $X$ be a set and let $\mathcal{X}$ be a family of subsets of $X$. Suppose that $\mathcal{T}, \mathcal{T}^{\prime} \in \mathcal{X}$ implies $\mathcal{T} \cap \mathcal{T}^{\prime} \in \mathcal{X}$. Then, we have $D(\mathcal{X})=\sigma(\mathcal{X})$.

This theorem implies the following lemma:

Lemma $5 \sigma(\mathcal{P}) \subseteq \mathcal{S}$.

Proof. Note that from the definition of $\mathcal{R}$, for any $\mathcal{T}, \mathcal{T}^{\prime} \in \mathcal{R}$, we have $\mathcal{T} \cap \mathcal{T}^{\prime} \in \mathcal{R}$. In addition, from Lemma $3, \mathcal{R} \subseteq \mathcal{S}$. From Dynkin's $\pi-\lambda$ theorem,

$$
\sigma(\mathcal{P})=\sigma(\mathcal{R})=\mathcal{D}(\mathcal{R}) \subseteq \mathcal{S}
$$

where the last set-inclusion holds from Lemma 4.

We are ready to show that, under Condition 3, the Shapley value and the prenucleolus coincide. In the proof, we use the following result proved by Kohlberg (1971):

Remark 2 (Kohlberg (1971)) Let $v \in \Gamma$ and $x \in \mathbb{R}^{n}$. Then, $x=\nu(v)$ if and only if for any $\alpha \in \mathbb{R},\{S \subseteq N, S \neq \emptyset: e(S, x, v) \geq \alpha\} \neq \emptyset$ implies that the family of coalitions is balanced.

Proposition 3 If $v \in \Gamma$ satisfies Condition 3 , then $\phi(v)=\nu(v)$.

Proof. Let $S \in 2^{N} \backslash \emptyset$ be fixed. We define $\mathcal{A}(S)$ by

$\mathcal{A}(S)=\left\{R \in 2^{N} \backslash \emptyset: R \in \mathcal{C}\left(T_{i}\right)\right.$ if and only if $S \in \mathcal{C}\left(T_{i}\right)$ for all $\left.i=1, \cdots, k\right\}$.

Then, from Lemma $5, \mathcal{A}(S)$ is a Shapley collection, especially, balanced. In addition,

$$
v^{S h}(S)=v^{S h}(R) \text { for all } R \in \mathcal{A}(S) \text {. }
$$

Then, for any $k \in \mathbb{R},\left\{T \in 2^{N}: v^{S h}(T) \geq k\right\} \neq \emptyset$ implies

$$
\left\{T \in 2^{N}: v^{S h}(T) \geq k\right\}=\cup_{T \in 2^{N} \backslash \emptyset: v^{S h}(T) \geq k} \mathcal{A}(T) .
$$

Since the union of balanced coalitions is again balanced, together with Remark 2, we have

$$
\nu\left(v^{S h}\right)=\mathbf{0}=\phi\left(v^{S h}\right) .
$$

From Weak Strategic Invariance,

$$
\phi(v)=\phi\left(v^{S h}\right)+\phi(v)=\nu\left(v^{S h}\right)+\phi(v)=\nu(v),
$$

which completes the proof. 
Suppose that a game $v$ satisfies Condition 3. Suppose also for any $T \in \mathcal{Q}(v)$, $|T|$ is an odd number. Then, from Propositions 1 and 2, the game $v$ is not symmetric, and does not satisfy PS property. However, from Proposition 3, $\phi(v)=\nu(v)$ holds. In this sense, new sufficient coincidence condition of this paper is described by Condition 3 .

\section{References}

[1] Chang, C., and Y. C. Tseng (2011): "On the coincidence property," Games and Economic Behavior, 71(2), 304-314.

[2] Chun, Y., and T. Hokari (2007): "On the coincidence of the Shapley value and the nucleolus in queueing problems," Seoul Journal of Economics, 20, $223-237$.

[3] Kar, A., M. Mitra and S. Mutuswami (2009): "On the coincidence of the prenucleolus and the Shapley value," Mathematical Social Sciences, 57(1), 16-25.

[4] Kohlberg, E. (1971): "On the nucleolus of a characteristic function game," SIAM journal on Applied Mathematics, 20, 62-66.

[5] Peleg, B., and P. Sudhölter (2007): Introduction to the theory of cooperative games, 2nd ed. Springer-Verlag.

[6] Schmeidler, D. (1969): "The nucleolus of a characteristic function game," SIAM Journal of Applied Mathematics, 17, 1163-1170.

[7] Shapley, L.S. (1953): "A value for n-person games," In: Roth AE (ed) The Shapley value. Cambridge University Press, Cambridge, pp 41-48.

[8] Yokote, K., Y. Funaki and Y. Kamijo (2013): "Linear basis approach to the Shapley value," Institute of Research in Contemporary Political and Economic Affairs Working Paper No.E1303, Waseda University. http://www.waseda-pse.jp/ircpea/jp/publish/working-paper-eseries/ Accessed 22 December 2014 


\section{Appendix}

Proof of Lemma 1. Let $T \subseteq N,|T| \geq 2, k \in \mathbb{N}, 2 \leq k \leq|T|$. We prove that the following equation holds:

$$
\bar{u}_{T}^{k}=\frac{1}{k}\left(\sum_{i \in T} \bar{u}_{T \backslash\{i\}}^{(k-1)}-(|T|-k+1) \bar{u}_{T}^{(k-1)}\right) .
$$

Let $S \subseteq N, S \neq \emptyset$. We calculate the worth of $S$ of both sides.

Case $10 \leq|T \cap S| \leq k-2$.

From the definition of $\bar{u}_{T}^{k}$, we have $\bar{u}_{T}^{k}(S)=\bar{u}_{T}^{(k-1)}(S)=0$. Consider the game $\bar{u}_{T \backslash\{i\}}^{(k-1)}, i \in T$.

$$
\begin{aligned}
& \text { If } i \in S,|(T \backslash\{i\}) \cap S| \leq k-3 . \\
& \text { If } i \notin S,|(T \backslash\{i\}) \cap S| \leq k-2 .
\end{aligned}
$$

It follows that $\bar{u}_{T \backslash\{i\}}^{(k-1)}(S)=0$ for all $i \in T$.

Case $2 k+1 \leq|T \cap S| \leq|T|$.

From the definition of $\bar{u}_{T}^{k}$, we have $\bar{u}_{T}^{k}(S)=\bar{u}_{T}^{(k-1)}(S)=0$. Consider the game $\bar{u}_{T \backslash\{i\}}^{(k-1)}, i \in T$.

$$
\begin{aligned}
& \text { If } i \in S,|(T \backslash\{i\}) \cap S| \geq k . \\
& \text { If } i \notin S,|(T \backslash\{i\}) \cap S| \geq k+1 .
\end{aligned}
$$

It follows that $\bar{u}_{T \backslash\{i\}}^{(k-1)}(S)=0$ for all $i \in T$.

Case $3|T \cap S|=k-1$.

From the definition of $\bar{u}_{T}^{k}$, we have $\bar{u}_{T}^{k}(S)=0$. Let $i \in T$.

$$
\begin{aligned}
& \text { If } i \in S,|(T \backslash\{i\}) \cap S|=k-2 . \\
& \text { If } i \notin S,|(T \backslash\{i\}) \cap S|=k-1 .
\end{aligned}
$$

That is, if $i \in S \cap T$, then $\bar{u}_{T \backslash\{i\}}^{(k-1)}(S)=0$. As a result,

$$
\begin{aligned}
\sum_{i \in T} \bar{u}_{T \backslash\{i\}}^{(k-1)}(S) & =\sum_{i \in T \backslash S} \bar{u}_{T \backslash\{i\}}^{(k-1)}(S) \\
& =\sum_{i \in T \backslash S} \bar{u}_{T}^{(k-1)}(S) \\
& =|T|-(k-1),
\end{aligned}
$$


where the second equality holds from the fact that $(T \backslash\{i\}) \cap S=T \cap S$ for $i \in T \backslash S$. Together with $-(|T|-k+1) \bar{u}_{T}^{(k-1)}(S)=-(|T|-k+1)$, the right-hand side is equal to 0 , which is equal to the left-hand side.

Case $4|T \cap S|=k$.

From the definition of $\bar{u}_{T}^{k}$, we have $\bar{u}_{T}^{(k-1)}(S)=0$. Let $i \in T$.

$$
\begin{aligned}
& \text { If } i \in S,|(T \backslash\{i\}) \cap S|=k-1, \\
& \text { If } i \notin S,|(T \backslash\{i\}) \cap S|=k .
\end{aligned}
$$

It follows that $\sum_{i \in T} \bar{u}_{T \backslash\{i\}}^{(k-1)}(S)=k$. By multiplying $\frac{1}{k}$, the right-hand side is equal to 1 , which is equal to the left-hand side.

Proof of Lemma 2. If $v$ satisfies PS property, as Kar et al. (2009) proved, we have

$$
\phi(v)=\nu(v)=\left(\frac{c_{1}}{2}, \cdots, \frac{c_{n}}{2}\right) .
$$

We know that $v^{S h}(N)=v^{S h}(\emptyset)=0$. We proceed by induction. Suppose that $v^{S h}(R)=v^{S h}(R)$ for all $R \subseteq N,|R|=r$, and we prove the result for $T \subseteq N,|T|=r+1$, where $r \geq 0$.

Let $i \in T$. Then,

$$
\begin{aligned}
v(T)-v(T \backslash\{i\})+v((N \backslash T) \cup\{i\})-v(N \backslash T) & =c_{i}, \\
v^{S h}(T)-v^{S h}(T \backslash\{i\})+v^{S h}((N \backslash T) \cup\{i\})-v^{S h}(N \backslash T) & =c_{i}-\frac{c_{i}}{2}-\frac{c_{i}}{2}=0 .
\end{aligned}
$$

From the induction hypothesis, $v^{S h}(T \backslash\{i\})=v^{S h}((N \backslash T) \cup\{i\})$. It follows that

$$
v^{S h}(T)=v^{S h}(N \backslash T) .
$$

Conversely, suppose that $v^{S h}(T)=v^{S h}(N \backslash T)$ for all $T \in 2^{N}$. Then, for any $T \in 2^{N}$,

$$
\begin{aligned}
v^{S h}(T)-v^{S h}(T \backslash\{i\})+v^{S h}((N \backslash T) \cup\{i\})-v^{S h}(N \backslash T) & =0, \\
v(T)-v(T \backslash\{i\})+v((N \backslash T) \cup\{i\})-v(N \backslash T) & =2 \phi_{i}(v),
\end{aligned}
$$

which means that PS property holds. 